\title{
ABSTRACT NO. \\ IGCS19-0311 \\ NEW EDUCATIONAL INTERVENTION WITH A SHORTER DURATION OF TRAINING PARAMEDICAL PROFESSIONALS (PMPs) FOR UNDERTAKING CERVICAL SCREENING PROGRAMME
}

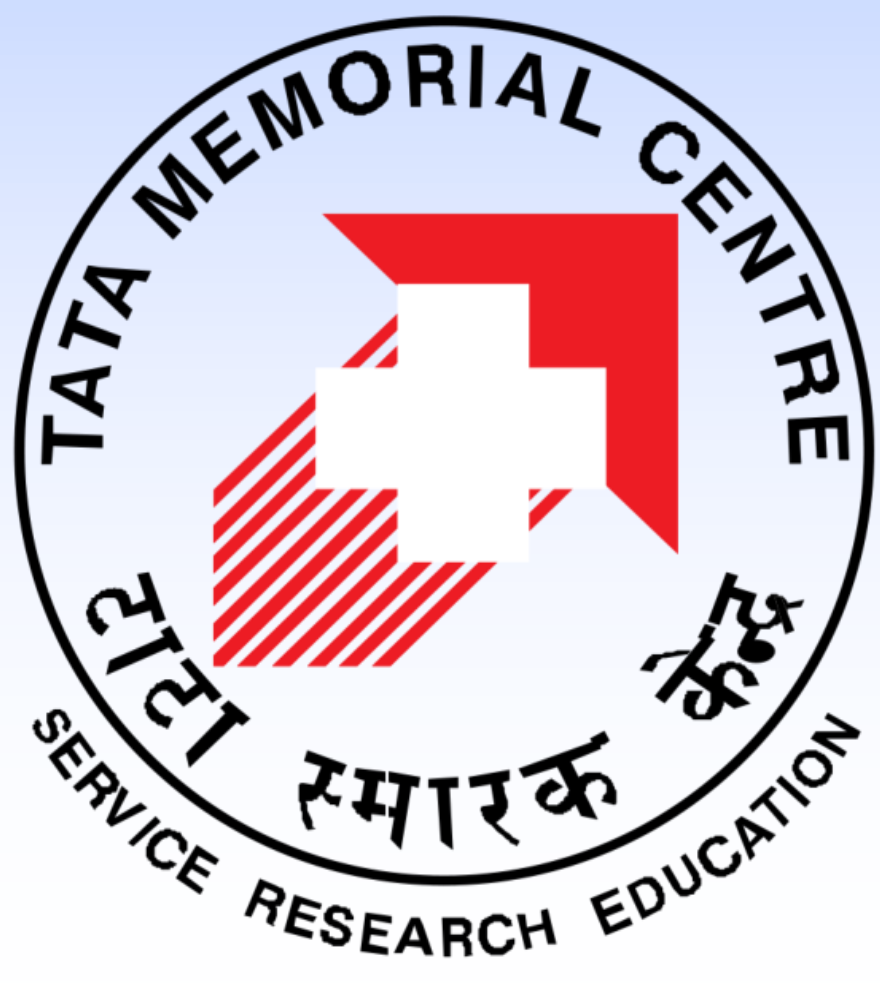

\author{
Shylasree TS, Misra G, Noronha S, Patil A, Kattepur A, Nandy P, Kulkarni V, Pramesh CS \\ Joint project of Tata Memorial Hospital and Indian Institute of Technology, Mumbai, India
}

\section{INTRODUCTION}

* Lack of awareness of disease and its prevention among general population and health workers together contributes to global burden of cervical cancer. * Capacity building by training of PMPs who are directly involved in community programmes is crucial to improve awareness and uptake of screening strategies.

* As PMPs were already working in other areas of community health care delivery, getting time off work for 6 weeks was logistically difficult. Hence reducing the training time was considered an alternative strategy without compromising on learning.

\section{AIMS}

To assess the effectiveness of a novel training programme (video based tutorial + hands on reduced duration of conventional training) for cervical cancer prevention among PMPs.

\section{METHODS}

$\checkmark$ Cross sectional analytical study. Fifty PMPs were included.

$\checkmark$ The novel training method consisted of video based tutorials, lectures and hands on training for 2 weeks.

$\checkmark$ PMPs were given personal electronic tablets consisting of video tutorials for revision and retention of knowledge during the training period. The tutorials consisted of health education and step by step practical methods of performing VIA/PAP smear/HPV testing . Preparation of Lugols iodine/acetic acid in primary health care center and other practical tips to improve specimen collection were included in the video.

$\checkmark$ Translations of the video tutorials were available in 3 languages English, Hindi and Marathi.

$\checkmark$ Questionnaires were administered before and after training programme.

$\checkmark$ Questionnaire consisted of 50 questions. There were 3 category of questions: multiselect, true/false and knowledge score.

$\checkmark$ These questions were further divided into 5 domains:

- awareness of cervical cancer

- awareness of cervical precancerous lesions

- practical screening methodology

- data management

- HPV and cervical cancer

$\checkmark$ Responses were scored by calculating the raw score and using linear transformation to standardize the raw score for all questions.

$\checkmark$ The raw score was calculated as follows:

Raw score (RS) = (sum of items of every question in each domain)/no. of question

$\checkmark$ Transformation score was calculated based on the category of questions:

- Multi-select questions: Total knowledge score $=[1-\{(\mathrm{RS}-1) /$ range $\}] \times 100$

- True/False questions: Total knowledge score $=[1-\{(\mathrm{RS}-1) /$ range $\}] \times 100$

- Knowledge based questions: Total knowledge score= [1-\{(RS-1)/range $\}]$ x100 $\checkmark$ A 3 point and a 5 point Likert scale was used for analysing the True/False and Knowledge based questions respectively.

\begin{tabular}{|c|c|c|c|c|c|}
\hline No. & DOMAINS & $\begin{array}{l}\text { TYPES OF } \\
\text { QUESTION }\end{array}$ & $\mathbf{n}$ & ITEM RANGE* & ITEM NUMBERS \\
\hline \multirow{2}{*}{1} & \multirow{2}{*}{$\begin{array}{l}\text { Cervical cancer } \\
\text { awareness }\end{array}$} & Multi-select & 2 & 3 & Q3\&Q6 \\
\hline & & True/False & 6 & 1 & Q2,Q4,Q5,Q7,Q8 \& Q9 \\
\hline \multirow[t]{3}{*}{2} & \multirow{3}{*}{$\begin{array}{c}\text { Cervical pre-cancer } \\
\text { awareness }\end{array}$} & \multirow{3}{*}{ Multi-select } & \multirow{3}{*}{3} & Range 3 for Q10 & Q10 \\
\hline & & & & Range 5 for Q12 & Q12 \\
\hline & & & & Range 7 for Q13 & Q13 \\
\hline \multirow{6}{*}{3} & \multirow{6}{*}{ Practice oriented } & \multirow{4}{*}{ Multi-select } & \multirow{4}{*}{12} & Range 4 for Q14 & Q14 \\
\hline & & & & \begin{tabular}{|c|} 
Range 3 for \\
Q15,Q16,Q21,Q22, Q23,Q30,Q \\
$32 \&$ Q37
\end{tabular} & 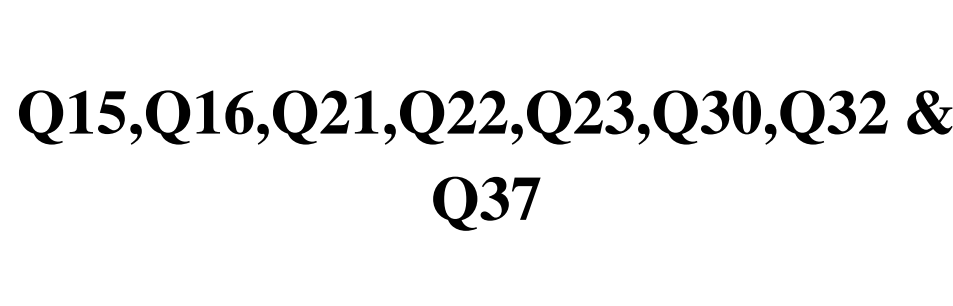 \\
\hline & & & & Range 7 for Q20 & Q20 \\
\hline & & & & Range 5 for Q24 \& Q34 & Q24 \& Q34 \\
\hline & & True/False & 8 & 1 & Q26,Q27,Q28,Q29,Q31 \& Q33 \\
\hline & & Knowledge & 5 & 4 & Q17g,Q17,Q18,Q19,Q25 \\
\hline \multirow[t]{2}{*}{4} & \multirow[t]{2}{*}{ Data management } & Multi-select & 2 & 3 & Q39,Q40 \\
\hline & & True/False & 1 & 1 & Q38 \\
\hline \multirow[t]{5}{*}{5} & \multirow[t]{5}{*}{ HPV/HPV vaccines } & \multirow[t]{3}{*}{ Multi-select } & 4 & Range 3 for Q42 \& Q50 & Q42 \& Q50 \\
\hline & & & & Range 7 for Q43 & Q43 \\
\hline & & & & Range 2 for Q45 & Q45 \\
\hline & & True/False & 3 & 1 & Q44,Q47\&Q49 \\
\hline & & Knowledge & 4 & 4 & Q11,Q41,Q46 \& Q48 \\
\hline
\end{tabular}

\section{RESULTS}

- Fifty PMPs participated in the novel training programme of 2 weeks duration.

- More than $90 \%$ of PMPs were married, parous and were from primary health care centers.

- Median work experience was 19.5 years

- Only $60 \%$ had themselves undergone cervical screening.

\begin{tabular}{|c|c|c|c|c|c|}
\hline & DOMAIN NAME & TYPE OF QUESTIONS & PRE-TEST & POST-TEST & p-VALUe \\
\hline \multirow[t]{2}{*}{1} & \multirow[t]{2}{*}{ Cervical cancer awareness } & Multi-select: median (IQR) & $\begin{array}{l}50.00 \\
(0.00)\end{array}$ & $\begin{array}{l}60.50 \\
(12.50)\end{array}$ & $<0.0001$ \\
\hline & & True/False: median (IQR) & $\begin{array}{l}75.00 \\
(8.33)\end{array}$ & $\begin{array}{l}91.67 \\
(8.33)\end{array}$ & $<0.0001$ \\
\hline 2 & Cervical pre-cancer awareness & Multi-select: mean \pm SD & $49.71 \pm 11.68$ & $67.41 \pm 15.71$ & $<0.0001$ \\
\hline \multirow{3}{*}{3} & \multirow{3}{*}{ Practice oriented } & Multi-select: mean \pm SD & $58 \pm 14.41$ & $76.6 \pm 8.43$ & $<0.0001$ \\
\hline & & True/False: mean \pm SD & $82.11 \pm 5.32$ & $92.46 \pm 3.02$ & $<0.0001$ \\
\hline & & $\begin{array}{l}\text { Knowledge score: median } \\
\text { (IQR) }\end{array}$ & $\begin{array}{c}70.00 \\
(35.00) \\
\end{array}$ & $\begin{array}{r}85.00 \\
(20.00)\end{array}$ & $<0.0001$ \\
\hline 4 & Data management & Multi-select: mean \pm SD & $49.25 \pm 27.71$ & $64 \pm 25.46$ & 0.001 \\
\hline \multirow{3}{*}{5} & \multirow{3}{*}{$\begin{array}{l}\text { Knowledge about HPV, } \\
\text { vaccination }\end{array}$} & Multi-select: mean \pm SD & $44.89 \pm 29.22$ & $77.28 \pm 11.45$ & $<0.0001$ \\
\hline & & True/False: median (IQR) & $\begin{array}{r}33.33 \\
(37.50) \\
\end{array}$ & $\begin{array}{c}100 \\
(16.67) \\
\end{array}$ & $<0.0001$ \\
\hline & & $\begin{array}{l}\text { Knowledge score: median } \\
\text { (IQR) }\end{array}$ & $\begin{array}{c}18.75 \\
(31.25)\end{array}$ & $\begin{array}{l}81.25 \\
(6.25)\end{array}$ & $<0.0001$ \\
\hline
\end{tabular}

Analysis showed very significant improvement in all domains after introduction of the novel training programme $(p<0.0001)$

\section{CONCLUSIONS}

The questionnaire survey was effective as it tested knowledge and awareness by multiple methods to improve response accuracy. The novel training programme is effective in significantly improving the training of PMPs with an added benefit of shorter duration of training. 